\title{
Especies de Fusarium en granos de maíz recién cosechado y desgranado en el campo en la región de Ciudad Serdán, Puebla
}

\author{
Fusarium species from corn kernels recently harvested and shelled in the fields in the Ciudad \\ Serdán Region, Puebla
}

Genoveva García-Aguirre* y Rebeca Martínez-Flores

Departamento de Botánica, Instituto de Biología. Universidad Nacional Autónoma de México. Apartado postal 70-233. 04510 México, D.F., México. *Correspondencia: gaag@servidor.unam.mx

\begin{abstract}
Resumen. Se analizaron 16 muestras de maíz, 10 de maíz blanco nacional y 6 de maíz criollo blanco para determinar las especies de Fusarium presentes en los granos, en especial aquellas registradas como inductoras de pudriciones de mazorca y grano, principalmente las que producen micotoxinas. Las especies identificadas en orden del número de aislamientos obtenidos fueron: Fusarium oxysporum, F. subglutinans, F. moniliforme, F. graminearum, F. anthophilum, F. poae, F. tricinctum, $F$. sporotrichioides y $F$. proliferatum. Con excepción de $F$. oxysporum, F. tricinctum y $F$. anthophilum, las demás han sido registradas como inductoras de pudriciones de mazorca, grano y de tallo, y la mayoría son productoras de diversas micotoxinas, algunas de las cuales pueden ocasionar problemas a la salud humana y animal.
\end{abstract}

Palabras clave: especies de Fusarium, maíz, pudriciones de mazorca y grano, micotoxinas.

\begin{abstract}
Sixteen corn samples, 10 of national white corn and 6 of "criollo" white were analyzed to determine the Fusaria species present on the kernels, especially those reported as ear and kernel rot inducers, mainly those mycotoxin producers. The identified species, ordered in relation to the number of obtained isolates were $F$. oxysporum, $F$. subglutinans, $F$. moniliforme, $F$. graminearum, $F$. anthophilum, F. poae, $F$. tricinctum, $F$. sporotrichioides, and $F$. proliferatum. The species F. oxysporum, F. tricinctum, and F. anthophilum have not been reported causing ear or kernel rots. All of the others have been reported as ear and kernel rots inducers, as well as stalk rots. Besides, most of these species produce various mycotoxins, many of which are capable of causing health problems to humans and animals.
\end{abstract}

Key words: Fusarium species, corn, ear and kernel rots, mycotoxins.

\section{Introducción}

En todas las regiones productoras de maíz del mundo, cada año se presentan enfermedades que afectan el rendimiento y la calidad del grano. En zonas húmedas, las pudriciones de mazorca son importantes, particularmente cuando la precipitación pluvial es mayor que la normal desde la época de la floración hasta la cosecha; en algunas regiones se han registrado daños severos causados por esas enfermedades. Entre las pudriciones de mazorca más relevantes están las inducidas por especies de Fusarium que además de reducir el rendimiento son causa del deterioro y mala calidad de los granos, y debido a la capacidad de producir micotoxinas también están relacionadas con enfermedades en humanos y en animales que los consumen (Charmley et al., 1994; Jelinek et al., 1989; Macías y Peraza, 2008; Shurtleff, 1980; Sutton,

Recibido: 25 abril 2008; aceptado: 15 junio 2009
1982; Tuite et al., 1974).

De las pudriciones de mazorca y de grano, las mejor conocidas son la llamada pudrición por Fusarium y la pudrición rosada de la mazorca inducida por Gibberella: Fusarium moniliforme J. Sheld. y Gibberella zeae (Schwein.) Petch= F. graminearum Schwabe., respectivamente. Estas especies, además de inducir pudriciones de mazorca, pueden producir diversas toxinas potencialmente riesgosas (Koehler, 1959; De León, 1974; Bacon y Williamson, 1992; Desjardins et al., 1994).

En México existe poca información reciente y accesible acerca de este tipo de pudriciones. Niederhauser (1949) registró a $F$. moniliforme como la causa más importante y más frecuente de las pudriciones en mazorca. Zenteno-Zevada (1963) y Medina (1979) aislaron mohos de mazorcas dañadas y encontraron que $F$. moniliforme fue la que se presentó con mayor incidencia. Además, existen registros de que esta especie ha sido aislada de mazorcas de maíz de los estados de Guanajuato, Guerrero, 
Hidalgo, Jalisco, Puebla y de Nuevo León, así como de F. graminearum, que fue aislada de mazorcas de maíz procedentes del Estado de México, Michoacán y Yucatán (Zenteno-Zevada, 1963, 1965; Zenteno-Zevada y Muñoz, 1968; Desjardins et al., 1994; Morales-Rodríguez et al., 2007).

El objetivo del presente trabajo fue conocer las especies de Fusarium en granos de maíz blanco procedentes de mazorcas recién cosechadas y desgranadas en el campo, en el centro oriente del estado de Puebla.

\section{Materiales y métodos}

Grano. Se obtuvieron 16 muestras de maíz, 10 de maíz blanco nacional y 6 de maíz criollo blanco, sembrado bajo condiciones de temporal, cada una de diferentes parcelas, la mayoría pequeñas, de 1 a 5 ha y de diferentes productores. La recolecta se realizó en 6 municipios de la región económica de Ciudad Serdán en el estado de Puebla; 5 muestras fueron de Ciudad Serdán, 4 de maíz blanco nacional y 1 de maíz criollo blanco; 5 de Tlachichuca y 1 de San Juan Atenco, de maíz blanco nacional; 2 de San Salvador el Seco, 2 de Aljojuca, y 1 de Soltepec, de maíz criollo blanco (Fig. 1).

Como en la zona muestreada no tienen secadores, cosechan el grano cuando el contenido de humedad es 13 a $16 \%$. El maíz fue desgranado en el campo y enviado inmediatamente al laboratorio, donde se determinó su contenido de humedad. Lo anterior, para que la micobiota presente en las mazorcas se modificara lo menos posible. Determinación del contenido de humedad. El contenido de humedad de los granos se determinó por triplicado para cada muestra, mediante el método de secado en estufa a $103^{\circ} \mathrm{C}$ por 72 horas (American Association of Cereal Chemist. 1962), se obtuvo por diferencia de peso y fue

Figura 1. Ubicación de los municipios muestreados.

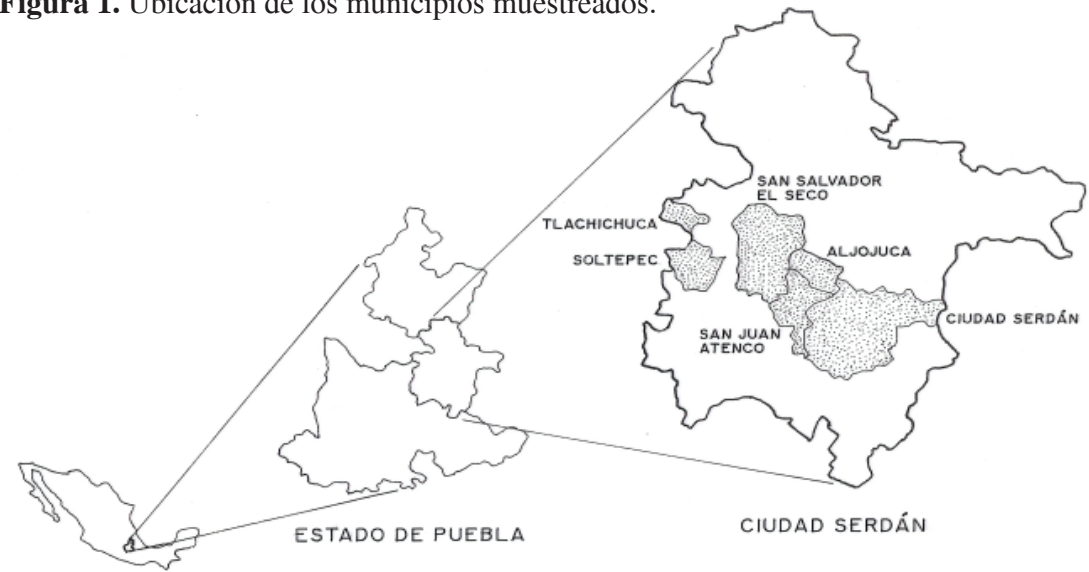

expresado con base en el peso húmedo.

Determinación de la micobiota. Se desinfectaron superficialmente 400 granos de maíz por muestra con hipoclorito de sodio al $1 \%$ durante un minuto, partiendo de una solución comercial al 5\%, y fueron sembrados en placas de Papa-Dextrosa-Agar (PDA) (International Seed Testing Association ISTA, 1993; Tuite, 1969), repartiendose 20 granos por placa y se incubaron a una temperatura de $25^{\circ} \mathrm{C} \pm 2^{\circ} \mathrm{C}$ por 7 días.

Identificación de hongos. Todos los aislamientos correspondientes al género Fusarium se identificaron hasta especie según Booth (1971) y Nelson et al. (1983). La incidencia de las especies fue expresada como frecuencia de los aislamientos (F) y densidad relativa (DR) de las especies, calculadas de acuerdo con la fórmula de Clarke y Christensen (1981).

Tratamiento estadístico. Además de la estimación de la frecuencia de los aislamientos y densidad relativa de las especies, se correlacionaron las variedades de maíz con la frecuencia de los aislamientos y $F$. graminearum y $F$. moniliforme con las localidades (Steel y Torrie, 1980).

\section{Resultados}

El contenido de humedad con el que el grano llegó al laboratorio fue de 11 a $13 \%$. El manejo del grano, desde la cosecha hasta su procesamiento en el laboratorio, fue hecho esperando que el número de aislamientos de Fusarium obtenidos fuese representativo del número de propágulos presente en la mazorca en el momento de la cosecha, ya que este hongo requiere de contenidos de humedad altos en el grano para incrementar sus poblaciones. Por otra parte, es de esperarse que los maíces criollos sean más resistentes a las pudriciones de mazorca y a otras enfermedades debido a su heterogeneidad genética. En el presente trabajo, dividiendo el número total de aislamientos entre el número de muestras de cada tipo de maíz se encontraron en promedio 311 aislamientos por muestra de maíz blanco nacional y 271 por muestra de maíz criollo blanco. Del maíz blanco nacional se obtuvieron 3114 aislamientos en total, que fueron identificados en 9 especies, y del maíz criollo blanco se aislaron 1629 mohos cuyas identificaciones correspondieron a 8 especies (Cuadro 1).

En relación con las localidades muestreadas, el mayor número de 
aislamientos se obtuvo en Tlachichuca con1627 mohos aislados de maíz blanco nacional, identificados en 8 especies de Fusarium. En Ciudad Serdán se obtuvieron 1210 aislamientos del maíz blanco nacional y 335 del criollo blanco, identificados en 6 especies; en San Salvador el Seco, 638 identificados en 8 especies; en Aljojuca, 441 identificados en 5 especies; en San Juan Atenco, 277 de maíz blanco nacional en 6 especies, y en Soltepec se obtuvieron 215 aislamientos en 3 especies (Cuadro1).

Con base en los coeficientes de correlación, podemos decir, con una probabilidad $\alpha .05$, que Fusarium crece igual en las 2 variedades de maíz estudiadas, asimismo, no existe diferencia en el crecimiento de F.graminearum y $F$. moniliforme en los diferentes municipios muestreados.

\section{Discusión}

Fusarium graminearum y $F$. moniliforme inducen pudriciones de mazorca y de tallo y tizón en las plántulas. En México, estos mohos están registrados como especies que inducen pudriciones de mazorca (Macías y Peraza, 2008, De León, 1974 y Zenteno-Zevada, 1963) y son de gran importancia por la capacidad que tienen para producir micotoxinas; entre las más importantes producidas por $F$. graminearum, sin. F. roseum Link (Tel. Gibberella zeae) están zearalenona, desoxinivalenol, diacetoxiscirpenol y zearalenol. Esta especie no fue aislada de las muestras, maíz criollo blanco, de Aljojuca. Las 2 parcelas muestreadas en esta localidad fueron de1 y 1.5 ha.

Fusarium moniliforme, sin. F. verticilloides (Sacc.) Nirenberg (Tel. Gibberella fujikuroi (Sawada) Wolenw. produce fumonisinas, ácido fusárico, fusarinas y naftoquinonas. Esta especie fue aislada de todas las muestras (Savard y Blackwell, 1994; Desjardins et al., 1994; Desjardins, 2006).

De Fusarium subglutinans (Wollenw. et Reinking) Nelson, Tousson et Marasas se obtuvieron 956 aislamientos; sin embargo, esta especie no fue aislada de la muestra proveniente de Soltepec, que fue de maíz criollo blanco. Es un hongo patógeno de la planta de maíz y componente importante de las pudriciones de mazorca en muchos lugares del mundo (Marasas et al., 1984; Desjardins, 2006).

En seguida se detallan algunas otras de las especies que presentaron una incidencia menor, y que también fueron identificadas. Fusarium poae (Peck) Wollenw., que se ha aislado como componente frecuente, pero generalmente de menor importancia, de pudriciones de mazorca por Fusarium y se le ha asociado con toxicosis en humanos y en animales (Logrieco et al., 2002; Desjardins, 2006). Fusarium sporotrichioides Sherb., asociada con pudriciones de mazorca en Estados Unidos de América, Canadá y Europa, se le relaciona con intoxicaciones en humanos y animales fue la especie dominante, aislada de los cereales dejados en el campo durante el invierno y que indujeron el brote de aleuquia alimentaria tóxica en 1940 en Rusia (Marasas, 1984; Beardall y Miller, 1994; Desjardins, 2006). Fusarium proliferatum (Matsush) Nirenberg ex Garlach et Nirenberg tiene un gran número de hospederos muy diversos en los que induce enfermedades con grandes daños para la economía; su incidencia en las pudriciones de mazorca cada día es mayor; no se le relaciona con intoxicaciones humanas (Logrieco et al., 2002; Desjardins, 2006). Fusarium oxysporum E.F. $\mathrm{Sm}$. et Swingle fue la especie que más frecuentemente se aisló de los 2 tipos de maíz en todas las localidades; es un hongo saprobio, ubicuo en el suelo, aislado de las raíces y semillas de muchas plantas, como parásito vegetal tiene varias formas especializadas que se conocen como formae specialis (f.sp.) que infectan diversas hospederas induciendo diferentes enfermedades; se le considera especie cosmopolita; sin embargo, las distribuciones de sus variadas formas especiales son más limitadas; tiene gran capacidad para producir toxinas de importancia económica y sanitaria (Davis y Diener, 1975; Thiel et al., 1992; Ueno; 1983, Savard y Blackwell, 1994). Fusarium tricinctum no se relaciona con toxicosis en humanos ni en animales, aunque es capaz de producir varias toxinas; por otra parte, en las regiones templadas se le ha relacionado con tizones en cereales, pero no con maíz, (Savard y Blackwell, 1994, Desjardins, 2006). De F. anthophilum no se obtuvieron aislamientos de las muestras de maíz criollo blanco de Aljojuca, Soltepec, donde las parcelas muestreadas eran de 1 ha en promedio; tampoco en las muestras de maíz blanco nacional de Ciudad Serdán, donde de las 2 parcelas fueron de 1 y de 5 ha. Llama la atención que se obtuviesen 123 aislamientos, ya que este hongo con muy poca frecuencia se aísla de suelos y de plantas, aunque puede encontrarse en gran diversidad de estas últimas. Poco se sabe de su biología, fitopatología y toxicología (Marasas et al., 1984; Desjardins, 2006).

A excepción de F. oxysporum, F. tricinctum (Corda) Sacc.y F. anthophilum (A. Brown) Wollenw. que no inducen pudriciones de mazorca, pero que pueden causar otras enfermedades en la planta en pie, el resto de las especies de Fusarium que se aislaron e identificaron están registradas como especies que inducen pudriciones de mazorca y que además producen diversas micotoxinas. Por lo que es necesario seguir haciendo este tipo de inspecciones de campo para garantizar la calidad del grano, ya que las pudriciones de mazorca ocasionan pérdidas económicas considerables y están relacionadas con enfermedades en animales y en humanos que consumen estos granos. 
กำ

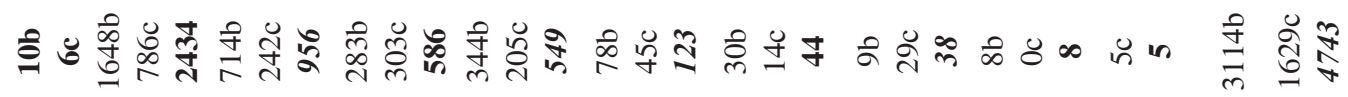

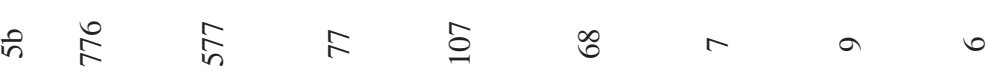

ิิ

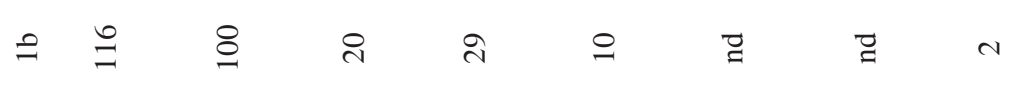

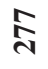

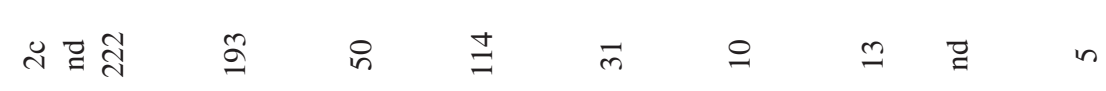

$\circ$ क

章

芯"

芯莡

$\stackrel{8}{\ddots}$

g

$\Rightarrow \quad \infty$

$\infty \quad$

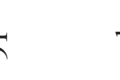

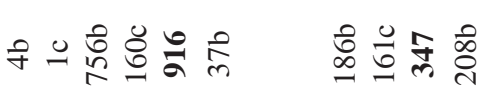

ป तิ ๘ี छ छ

을 穴

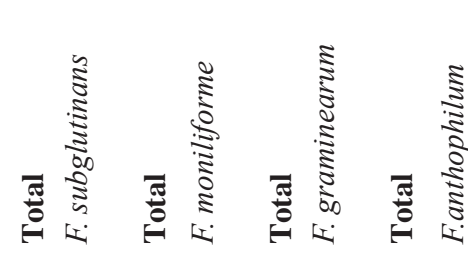

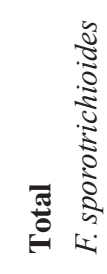

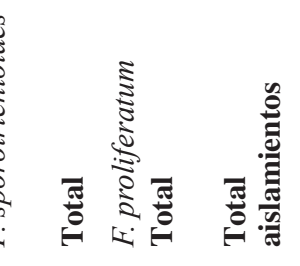


Lo anterior resulta particularmente importante en localidades con índices de marginación altos, como las que se encuentran en Tlachichuca, Aljojuca, San Juan Atenco y Soltepec, 4 de los municipios donde se realizó este estudio, que ocupan los lugares de marginación 127,131, 140 y 148, respectivamente, entre los 217 municipios del estado, en los que se acentúan los efectos de la pobreza, como la desnutrición, los bajos niveles de escolaridad, altaincidencia de enfermedades e ífima calidad de la vivienda, y San Salvador el Seco y Chalchicomula (Ciudad Serdán) que han sido calificados con niveles de marginación medios, ocupan los lugares 171 y 179 respectivamente. Los porcentajes de mortalidad infantil son de 30.3, 26.3, 27.8, 40, 84.2 y $46.5 \%$, y sus poblaciones se dedican, económicamente, al sector primario (agricultura, ganadería, caza y pesca) 82.7, 79.8, 85, 82.7, 51.9 y 59.3\%. Aunque en San Salvador el Seco y Chalchicomula existen zonas de riego, el tipo de agricultura que se practica en estos municipios es de temporal y principalmente, dado el tamaño y rendimiento de sus parcelas, de autoconsumo. La ganadería en estos municipios es de traspatio, con excepción de Soltepec que produce ganado porcino en grandes cantidades. Según el Consejo Nacional de Evaluación, el estado de Puebla ocupa el sexto lugar en pobreza alimentaria (Enciclopedia de los Municipios, Puebla, 2009).

Lo precedente implica que los porcentajes de pérdidas causadas por Fusarium en parcelas del tamaño de las estudiadas comparadas con las de más de 50 ha pueden llegara a ser devastadores. Asimismo, el riesgo sanitario para esas poblaciones deprimidas y mal alimentadas puede ser alto y seguramente no se ha detectado o ha sido encubierto por otras enfermedades.

También es importante la identificación correcta de las especies. F. tricinctum y F. anthophilum, que aparentemente fueron mal clasificadas en el pasado y probablemente se modifiquen sus cualidades fitopatógenas y toxicológicas (Desjardins, 2006).

\section{Literatura citada}

American Association of Cereal Chemist. 1962. Cereal laboratory methods, $7^{\text {th }}$ edition. St. Paul, Minnesota.

Bacon, W. C. y W. J. Williamson.1992. Interactions of Fusarium moniliforme, its metabolites and bacteria with corn. Mycopathologia 117:65-71.

Beardall, J. M. y J. D. Miller. 1994. Diseases in humans with mycotoxins as possible causes. In Mycotoxins in grain: compounds other than aflatoxin, J. D. Miller y H. L.

Trenholm (eds.). Eagan, Minnesota. p. 487-539.

Booth, C. 1971. The genus Fusarium. Commonwealth Mycological Institute, Kew, Surrey. 237 p.

Charmley, L. L., A. Rosenberg y L. H. Trenholm. 1994. Factors responsible for economic losses due to Fusarium mycotoxin contamination of grains, food, and feedstuffs. In Mycotoxins in grain: compounds other than aflatoxin, D. J. Miller y L. H. Trenholm (eds.). Eagan, Minnesota. p. 471-486.

Clarke, D. C. y M. Christensen.1981. The soil microfungal community of a South Dakota grassland. Canadian Journal of Botany 59:1950-1960.

Davis, N. D. y V. L Diener. 1975. Mycotoxins. In Food and beverage mycology, L. R Beauchat (ed.). Avi, Westport, Connecticut. p. 397-444.

De León, C. 1974. Enfermedades del maíz. Guía para su identificación en el campo. Folleto Técnico de Información 11, Centro Internacional de Mejoramiento de Maíz y Trigo (CIMMyT), México, D.F. 77 p.

Desjardins, A. E., D. R. Plattner y P. E. Nelson. 1994. Fumonisin production and other traits of Fusarium moniliforme strains from maize in northeast Mexico. Applied and Environmental Microbiology 60:1695-1697.

Desjardins, A. E. 2006. Fusarium mycotoxins. chemistry, genetics, and biology. The American Phytopathological Society, St. Paul, Minnesota. 260 p.

Enciclopedia de los Municipios de México, Puebla. 2009. www. e-local.gob.mx/work/temptales/enciclo/puebla/index.html; 12.01.2009.

Jelinek, C. F., A. E. Pohland y G. E. Wood. 1997. Review of mycotoxin contamination. Journal Association Official Analytical Chemistry 72:223-230.

International Seed Testing Association. 1993. Seed health testing. Seed Science \& Technology 21:33-36.

Koehler, B. 1959. Corn ear rots in Illinois. III. Agricultural Experiment Station Bulletin $639: 87$.

Logrieco, A., G. Mulè, A. Moretti y A. Bottalico. 2002. Toxigenic Fusarium species and mycotoxins associated with maize ear rot in Europe. European Journal of Plant Pathology 108:597-609.

Macías Cervantes, J. y S. Peraza Medina. 2008. Enfermedades del maíz en el norte de Sinaloa. Agronet. www.agronet.com. $\mathrm{mx} /$ articles.cgi? Action=Viewhistory $\&$ Article $=3 \&$ Type $=\mathrm{A}$; 31.03-2008.

Marasas, W. F. O-, P. E. Nelson y T. A. Toussoun. 1984. Toxigenic Fusarium Species, Identity and Mycotoxicology. The Pennsylvania State University. 328 p.

Medina C. D.1970. Pudriciones fungosas en mazorcas de maíz (Zea mays L.). Tesis, Facultad de Ciencias, Universidad Nacional Autónoma de México. México, D. F. 23 p.

Morales-Rodríguez. I., M. J. Yañez-Morales, H. V. Silva-Rojas, G. García-de-los-Santos y D. A. Guzmán-de-Peña. 2007. Biodiversity of Fusarium species in Mexico associated with ear rot in maize, and their identification using a phylogenetic approach. Mycopathologia 163:31-39.

Nelson, P. E., T. A. Toussoun y W. F. O. Marasas. 1983. Fusarium species: An illustrated manual for identification. The Pennsylvania State University Press, University Park p. 193.

Neiderhauser, J. S. 1949. Enfermedades del maíz en México, Folleto de divulgación 9. Secretaría de Agricultura y Ganadería, México, D.F. 39 p.

Savard, M. E. y B. A. Blackwell.1994. Spectral characteristics of secondary metabolites from Fusarium fungi. In Mycotoxins in grain: compounds other than aflatoxin, J. D. Miller and 
H.L. Trenholm (eds.). Eagan, St. Paul, Minnesota. p. 59-69. Shurtleff, M. C. 1980. Compendium of corn diseases. American Phytopathological Society, St. Paul, Minnesota. 105 p.

Steel R.G.D. y .H. Torrie. 1980. Principles and procedures of statistics. A biometrical approach. McGraw-Hill, New York.633 p.

Sutton, J. C. 1982. Epidemiology of wheat head blight and maize ear rot caused by Fusarium graminearum. Canadian Journal Plant Pathology 4:195-209.

Thiel, P. G., W. F. O. Marasas, E. W. Sydenham., G. S. Shephard y W. C. Gelderblom. 1992. The implications of naturally occurring levels of fumonisins in corn for human and animal health. Mycopathologia 117: 3-9.

Tuite, J. 1969. Plant pathological methods: fungi and bacteria. Burguess, Minneapolis, Minnesota. 239 p.

Tuite, J., G. Sharner, G. Rambo, J. Foster y R.W. Caldwell. 1974. The Gibberella ear rot epidemics of corn in Indiana in 1965 and 1972. Cereal Science Today 19: 238-241.
Ueno, Y. 1983. Trichothecenes. Chemical, Biological and Toxicological Aspects. Elsevier, Amsterdan. p.313.

Zenteno-Zevada, M. 1963. Estudios sobre hongos parásitos de gramíneas de la República Mexicana. III. Pruebas de inoculación en plántulas de maíz con Gibberella fujikuroi (Saw.) Wr. Anales del Instituto de Biología, Universidad Nacional Autónoma de México 34:69-83.

Zenteno-Zevada, M. 1965. Estudios sobre hongos parásitos de gramíneas de la República Mexicana. IV. Pruebas de inoculación en mazorcas de maíz con Fusarium moniliforme Sheld. Wr. Anales del Instituto de Biología, Universidad Autónoma de México, Serie Botánica 34:69-78

Zenteno-Zevada, M. y O. A. Muñoz. 1968. Estudios sobre hongos parásitos de gramíneas de la República Mexicana. VII. Segunda prueba de inoculación en mazorcas de maíz con Fusarium moniliforme Sheld. Anales del Instituto de Biología, Universidad Nacional Autónoma de México, Serie Botánica 34:111-116. 